\title{
Detection of Dentin Matrix Protein 1 and Osteopontin in Tertiary Dentine During Experimental Extrusion in Rat Incisors Revealed by
} Immunocytochemistry

M.C. Aguiar, * V.E. Arana-Chavez **

* Department of Biomorphology, Institute of Health Sciences, Federal University of Bahia, Salvador, BA, Brazil

** Division of Oral Biology, School of Dentistry, University of São Paulo, São Paulo, SP, Brazil.

Although the main mechanisms of primary dentinogenesis are reasonably understood, several aspects regarding tertiary dentine formation are still matter of investigation. Some of these aspects may be related with specific noncollagenous proteins elaborated by specialized cells. As dentine matrix protein 1 (DMP1) is present in primary dentine, it is possible that this noncollagenous protein may be also present in the matrix secreted after tissue injury, but there are no studies attempting to detect it in tertiary dentine. The aim of the present study was to examine the ultrastructural immunolocalization of DMP1 in the tertiary dentine after intentional extrusion of the rat incisor, comparing with distribution of osteopontin (OPN). Forty-nine male Wistar rats were divided into two groups. The first (experimental) group included 28 animals whose upper right incisor was extruded $3 \mathrm{~mm}$ and then repositioned into its original socket. The second (control) group included 21 animals whose incisors were not extruded. At 3, 7, 10, 15, 20, 30 and 60 days after surgery, the incisors were fixed and then processed for scanning and transmission electron microscopy and for immunocytochemistry for DMP1 and OPN. As established in our previous study, the pulp trauma model provoked formation of reparative dentine with variable aspect and related cells (Aguiar and Arana-Chavez 2007). DMP1 was present in the extracellular matrix of all types of dentine, i.e., physiologic, reactionary, and reparative, always in association with collagen fibrils found in the entire dentine matrix, but rare colloidal gold particles over predentine (Fig 1A). DMP1 was evident in the supranuclear region and inside the nucleus of some odontoblast-like cells (Fig. 1B). DMP1 was too diffusely distributed into collagen fibrils of the reparative dentine matrix near to cellular debris (Fig. 1C). OPN was only detected in the reparative dentine, being strongly immunoreactive within some interfacial structures (Fig. 1D) These findings showed association between DMP1 and collagen seem to be essential for dentine formation, besides this protein may moves to nucleus for playing a role in the regulation of genes that control the odontoblast differentiation, as showed in original odontoblasts during early dentinogenesis (Massa et al. 2005) and in odontoblast-like cells during in vitro experiments (Narayanan et al. 2001).

\section{References}

[1] M.C. Aguiar, V.E Arana-Chavez, J. Anat. 210 (2007) 418-427

[2] L.F. Massa et al. Histochem. Cell Biol. 124 (2005) 197-205

[3] K. Narayanan et al. Proc. Natl Acad. Sc.i USA 98 (2001) 4516-4521

[4] This work was partially supported by CNPq (Brazil). 

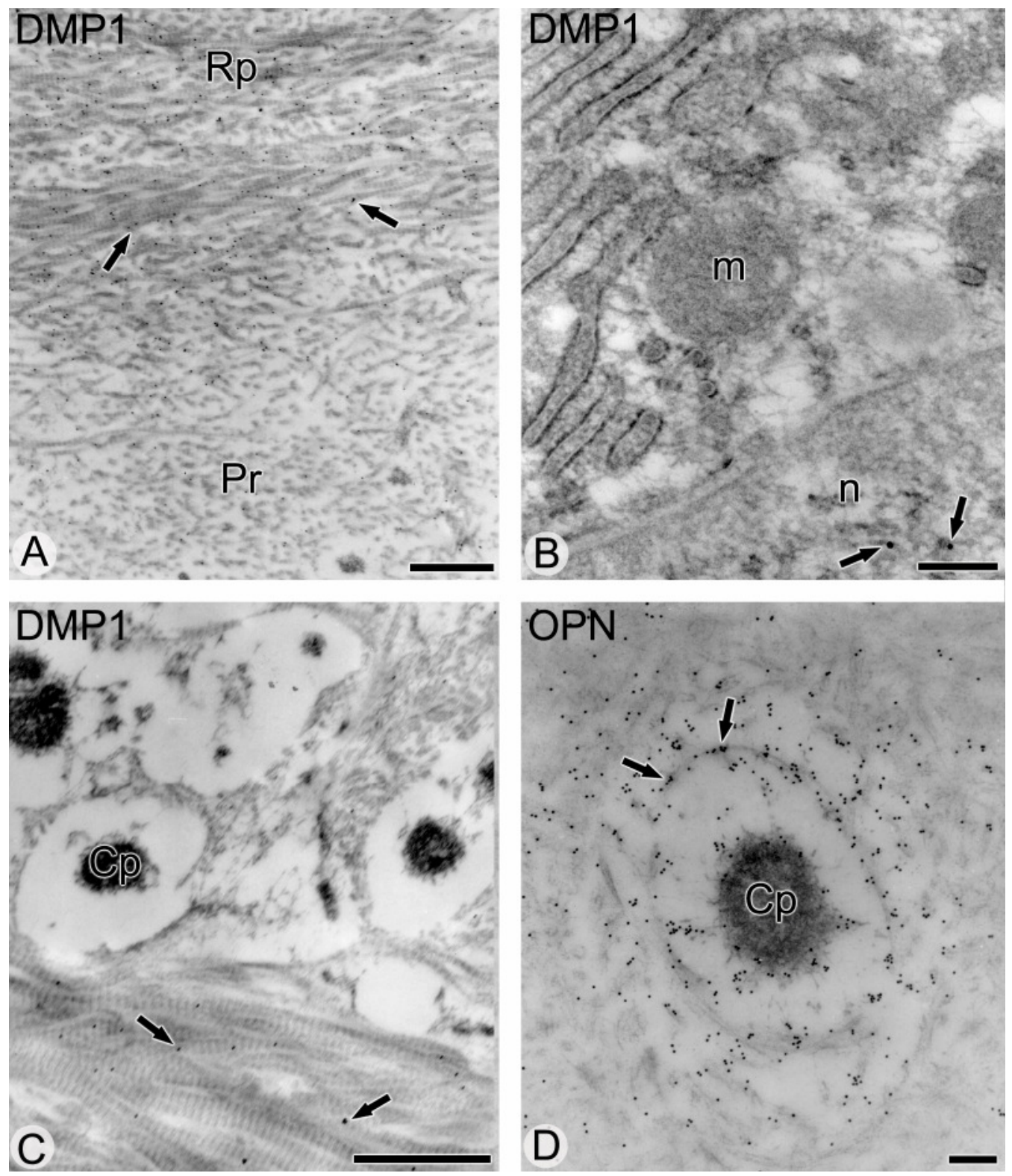

Fig. 1. Transmission electron micrographs showing several regions of extruded rat incisors processed for postembedding colloidal gold immunocytochemistry for DMP1 and OPN. In A, an area of reparative dentine (Rp) exhibiting gold particles immunoreactive for DMP1 (arrows). Note that predentine (Pr) displays few labeling for DMP1. Scale bar=1 $\mu \mathrm{m}$. In $\mathbf{B}$, odontoblast-like cell showing labeling for DMP1 (arrows) within the nucleus (n). Note that a mitochondrion (m) is free of gold particles. Scale bar $=0,2 \mu \mathrm{m}$. In $\mathbf{C}$, some gold particles for DMP1 are diffusely distributed into collagen fibrils of the whole reparative dentine matrix, including in the regions near to cellular debris (arrows). Scale bar $=1 \mu \mathrm{m}$. In $\mathbf{D}$, gold particles immunoreactive for OPN forming patches (arrows) appear in the reparative dentine matrix in close relation to cellular debris. Scale bar $=1 \mu \mathrm{m}$. 